\title{
Messung der Zusammensetzung von 2-Phasenmischungen mittels Ringelektroden
}

\author{
Herbert Bousack ${ }^{1}$, Manu Mohan ${ }^{1}$, Ran Zhou ${ }^{1}$, Kavita Mayekar ${ }^{2}$ und Gerhard von der Emde ${ }^{2}$ \\ Forschungszentrum Jülich, Peter-Grünberg-Institut PGI-8, Jülich, Deutschland \\ h.bousack@fz-juelich.de \\ ${ }^{2}$ Universität Bonn, Institut für Zoologie, Bonn, Deutschland
}

\begin{abstract}
:
Mehrphasenströmungen treten in der Verfahrenstechnik sehr häufig auf, z.B. Mischungen von Gas und Flüssigkeit, von Feststoffen und Flüssigkeiten oder Flüssigkeitsmischungen. Für eine zuverlässige Prozesssteuerung müssen die Strömungsparameter, wie z.B. die Feststoffkonzentration, der Gasanteil oder die Größe und Geschwindigkeit von Blasen, einfach und zuverlässig gemessen werden. Mit einem Verfahren, das teilweise auf dem biomimetischen Verfahren der Elektrolokation der schwache elektrischen Elefantenrüsselfische Gnathonemus petersii beruht, konnte gezeigt werden, dass die Mischung von Flüssigkeiten mit unterschiedlichen elektrischen Leitfähigkeiten, z.B. Wasser und Ethanol, und Blasenströme von Luft in Wasser gut detektiert werden konnten. Hierbei ergibt sich, insbesondere bei Flüssigkeitsmischungen, eine lineare Abhängigkeit des Sensorsignals vom Volumenanteil der Zumischung bzw. vom Volumenstrom der Blasen.
\end{abstract}

Key words: Bionische Sensoren, schwach elektrischer Fisch, Mehrphasenströmungen, elektrische Leitfähigkeit, Messung von Mischungsanteilen

\section{Einleitung}

Eine Mehrphasenströmung wird als die gleichzeitige Strömung einer Mischung von zwei oder mehreren Phasen flüssig / gasförmig / fest definiert [1]. Sie tritt in der Verfahrenstechnik sehr häufig auf, z.B. Mischungen von Gas und Flüssigkeit in Blasensäulen, Mischungen von Feststoff und Flüssigkeit bei der Feststoffförderung oder auch Flüssigkeitsmischungen. Die Messung der Feststoffkonzentration, des Gasanteils oder der Größe und Geschwindigkeit von Blasen benötigt eine zuverlässige Sensorik zur Prozesssteuerung. Neben optischen Messungen [2], Ultraschall[3] oder Röntgen-Methoden [4] werden sehr oft Verfahren verwendet, die auf der Änderung der elektrischen Leitfähigkeit [5], [6] oder der kapazitiven Eigenschaften [7], [8] beruhen. Die sogenannten Gasgehalt (void fraction) Sensoren oder die elektrische Impedanztomographie (EIT) sind jedoch teilweise weniger gut geeignet wegen zu geringer Genauigkeit bei der Messung sehr kleiner Anteile, z. B. bei Gasvolumenanteilen kleiner als $5 \cdot 10^{-4}$ [3], oder zu aufwändiger Technologie. Für die Prozessregelung wäre aber eine einfache und genaue Messtechnik wünschenswert.

Auch in der Medizintechnik wird die zuverlässige Detektion von Gasblasen oder des Gasanteils in Flüssigkeiten häufig benötigt, z.B. die
Erkennung von Luftblasen bei der Dialyse oder bei Infusionen. Hierbei werden verschiedenen Messprinzipien eingesetzt, z.B. Ultraschall- [9] , kapazitive [10], thermische [11] oder Impedanz[12] Verfahren.

Im Folgenden wird ein neuartiges Verfahren vorgestellt, das im Wesentlichen auf der aktiven Elektroortung von schwach elektrischen Fischen beruht und damit in den Bereich der Impedanz-Messverfahren eingeordnet werden kann. Schwach elektrische Fische, z.B. der Elefantenrüsselfisch Gnathonemus petersii, erkunden ihre Umwelt durch die Erzeugung eines sie umgebenden elektrischen Feldes, das sie als pulsförmige Entladung im Stiel ihrer Schwanzflosse erzeugen. Zur Registrierung des elektrischen Felds besitzen sie bis zu 3000 Elektrorezeptoren, die auf ihrer Haut - insbesondere im Kopfbereich - verteilt sind. Fremdkörper im Bereich des elektrischen Felds verändern den Verlauf der Feldlinien und können so mit den Elektrorezeptoren registriert werden [13]. Damit ist es den Fischen möglich, selbst in vollständiger Dunkelheit Beute zu finden und Gegenständen bezüglich ihrer Größe, Form und Materialeigenschaft zu klassifizieren [14]. Dieses Prinzip wurde schon bei der Entwicklung von Herz-Kathetern eingesetzt mit dem Ziel, Plaque-Ablagerungen in den Blutgefäßen zu erkennen und vermessen. Hierbei 
wird mit einer Sendeelektrode im Katheter ein elektrisches Feld erzeugt und die Feldänderungen werden mit einem Array von ebenfalls auf dem Katheter angeordneten Empfangselektroden ausgelesen [15]. Bei dieser Anwendung werden der Katheter und damit die Elektroden bewegt, um ein stationäres Objekt zu finden und zu analysieren. Man kann dieses Prinzip aber auch verwenden, wenn die Elektroden stationär angeordnet werden und sich das Objekt bewegt [16]. Bei dieser Anwendung können die Elektroden z.B. als Ringe ausgebildet werden und das zu lokalisierende Objekt kann eine Luftblase im Wasser sein, die sich mittels Auftrieb oder erzwungener Strömung durch den Ring bewegt. Grundsätzlich können Objekte gemessen werden, wenn die elektrischen Leitfähigkeiten von Objekt und Fluid hinreichend unterschiedlich sind. Der Unterschied dieses Verfahrens im Vergleich zur Prozess-Tomographie [5], die durch Reduzierung der Elektroden zu einem ähnlichen Ansatz führt, besteht in der Signalauswertung, bei der vergleichbare Ansätze wie bei den schwach elektrischen Fischen verfolgt werden.

\section{Messverfahren}

Das grundsätzliche Messverfahren, im folgenden Blasen-Detektor genannt, ist in Bild 1 dargestellt. Mit zwei ringförmigen Elektroden, einer Sende- und einer Empfangselektrode axial in einem Rohr mit einem Innendurchmeser von $40 \mathrm{~mm}$ angeordnet, wird in einem elektrisch leitfähigen Fluid, z.B. Wasser, ein Feld erzeugt und ausgelesen, wobei Objekte, die in das Feld eindringen, den Verlauf der Potentialfeldlinien zwischen den Elektroden verändern. Die Sendeelektrode wird mit einem Sinusgenerator (z.B. $1 \mathrm{~V}, 1 \mathrm{kHz}$ ) verbunden und der an der Empfangselektrode abgeführte Strom wird über einen regelbaren Shunt-Widerstand im $\mathrm{k} \Omega$ Bereich an einen Differenzverstärker angeschlossen, der die Spannungsdifferenz aus der Sendeelektrode und der Empfangselektrode verstärkt. Eine Kompensationseinstellung sorgt dafür, dass das Sendesignal entsprechend reduziert wird (siehe Bild 1). Nach dem Differenzverstärker wird das Signal mit einem Lock-in-Verstärker als schmalbandigen Bandpassfilter auf der Sendefrequenz verstärkt und angezeigt.

\section{Maxwell-Modell zur Berechnung des Mischungsanteils}

Zur Berechung des Mischungsanteils in einem Volumen sowie in einem durchströmten Behälter aus den elektrischen Leitfähigkeiten der Fluide wird oft die Maxwell - Gleichung [17] verwendet, die von einem Modell von vielen

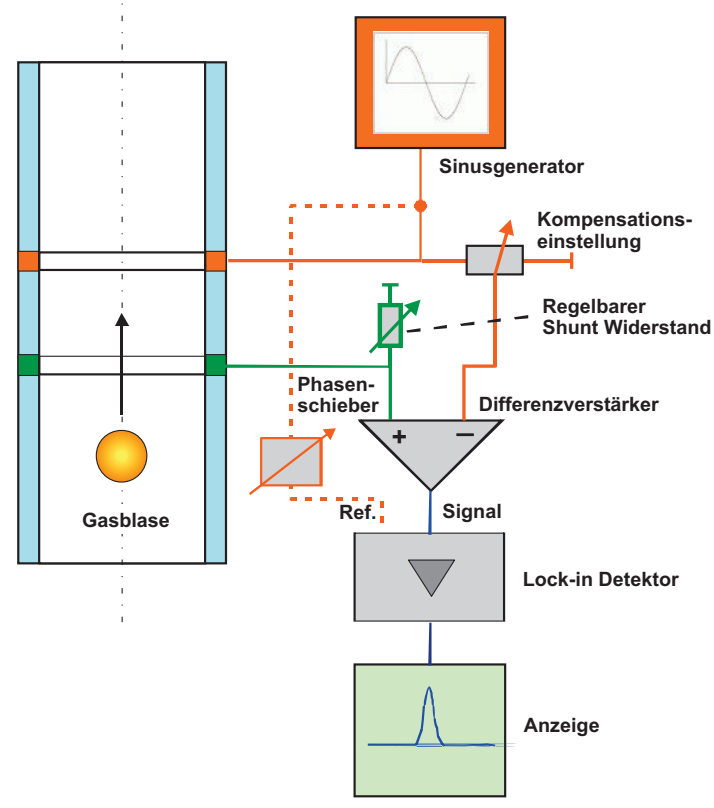

Bild 1. Prinzip des Messverfahrens

kleinen Kugeln mit der elektrischen Leitfähigkeit $\sigma_{Z}$ (Z, Zumischung) innerhalb einer großen Kugel mit der Leitfähigkeit $\sigma_{T} \quad(T$, Trägermaterial) ausgeht.

$\Phi_{Z}=\frac{\sigma_{\mathrm{Z}}+2 \cdot \sigma_{\mathrm{T}}}{\sigma_{\mathrm{Z}}-\sigma_{\mathrm{T}}} \cdot \frac{\sigma_{\mathrm{Mix}}-\sigma_{\mathrm{T}}}{\sigma_{\mathrm{Mix}}+2 \cdot \sigma_{\mathrm{T}}} \quad \Phi_{\mathrm{Z}}+\Phi_{\mathrm{T}}=1$

Mit: $\phi_{\mathrm{Z}}, \phi_{\mathrm{T}}$ : Anteil der Komponenten der Zumischung bzw. des Trägermaterials, $\sigma_{Z}, \sigma_{T}$ : elektrische Leitfähigkeiten der Zumischung bzw. des Trägermaterials, $\sigma_{\text {Mix: }}$ elektrische Leitfähigkeit der Mischung Komponenten Z und $\mathrm{T}$.

Wenn die Leitfähigkeit der Zumischung wesentlich kleiner ist als die Leitfähigkeit des Trägermaterials, wie es z. B. für die Mischung von Luft und Wasser zutrifft, dann ergibt sich aus GI. 1 die Maxwell-Hewitt Gleichung [18], [19]. Eine wichtige Voraussetzung ist hierbei, dass beide Komponenten z. B. bei einer Rohrströmung die gleiche Strömungsgeschwindigkeit aufweisen.

$$
\Phi_{\mathrm{Z}}=\frac{1-\left[\frac{\sigma_{\mathrm{Mix}}}{\sigma_{\mathrm{T}}}\right]}{1+\lambda \cdot\left[\frac{\sigma_{\mathrm{Mix}}}{\sigma_{\mathrm{T}}}\right]}
$$

Mit: $\lambda$ : Geometriefaktor. $\lambda=0,5$ für kugelförmige Einschlüsse (Zumischung) für $\sigma_{Z}<<\sigma_{T}$ [17], $\lambda=$ 0,9 - 0,25 für ellipsoide Einschlüsse in Abhängigkeit des Hauptachsenverhältnisses, der Ausrichtung und dem Verhältnis von $\sigma_{Z}$ und $\sigma_{\mathrm{T}}[20], \quad \lambda=1$ für zweidimensionale Einschlüsse [19]. 
Alternative zu Gleichung (1) und (2) wurden modifizierte Ansätze entwickelt, z.B. das DipolModell [21], die Bruggeman- [22] oder die Botcher-Gleichung [23]. Das Bild 2 zeigt einen Vergleich dieser Gleichungen mit der MaxwellHewitt Gleichung.

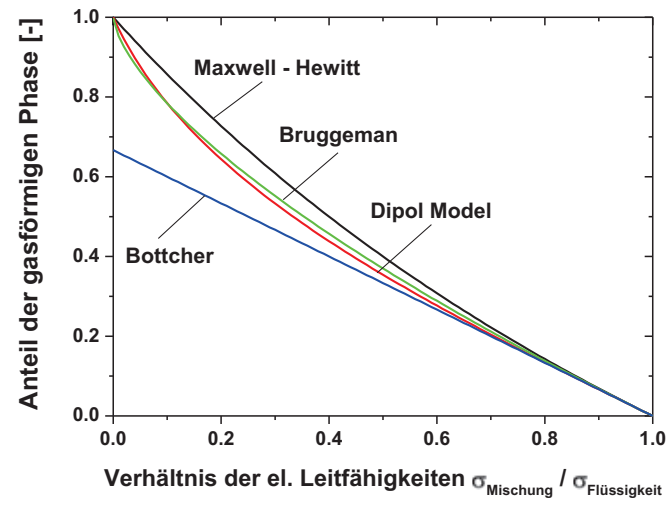

Bild 2. Vergleich verschiedener Gleichungen zur Bestimmung des Mischungsanteils der gasförmigen Phase aus dem Verhältnis der elektrischen Leitfähigkeiten der Mischung und der flüssigen Phase

Für Zumischungsanteile kleiner als 0,2 stimmen alle Gleichungen gut überein, erst bei größeren Zumischungsanteilen ergeben sich deutliche Differenzen. Nach [21] wird die Verwendung der Maxwell-Hewitt Gleichung oder das DipolModell empfohlen.

Die Gleichung (2) ist signifikant abhängig von der Geometrie der Einschlüsse und sollte gute Ergebnisse bei z.B. kleinen Gasblasen als kugelförmige Einschlüsse zeigen. Für die Mischung von zwei Flüssigkeiten wurde in der Literatur kein Geometriefaktor gefunden. Zur Abschätzung des Geometriefaktors für diesen Fall wurde in ein Wasservolumen $\left(\sigma_{T}=100,4\right.$ $\left.\mu \mathrm{S} / \mathrm{cm}, \quad 28{ }^{\circ} \mathrm{C}\right)$ jeweils Ethanol $\left(\sigma_{z}=0,94\right.$ $\mu \mathrm{S} / \mathrm{cm}, 28^{\circ} \mathrm{C}$ ) zugemischt und die Leitfähigkeit gemessen (Mettler Toledo, SevenEasy Conductivity Meter S30). Das EthanolVolumenverhältnis in der Mischung wurde berechnet und mit dem mit der Gleichung (2) berechneten Volumenverhältnis verglichen, siehe Bild 3.

Aus Bild 3 ergibt sich, dass mit einem Geometriefaktor von $\lambda=1,3$ die Gleichung (2) den Volumenanteil Ethanol bis etwa $35 \%$ gut wiedergibt. Allerdings ist der Geometriefaktor abhängig von den Leitfähigkeiten der Komponenten, so ergibt sich z.B. für ein Verhältnis von $\sigma_{Z} / \sigma_{T}=0,07$ ein $\lambda=1,9$ mit einer guten Übereinstimmung des gerechneten und gemessenen Volumenanteils nur bis $8 \%$.

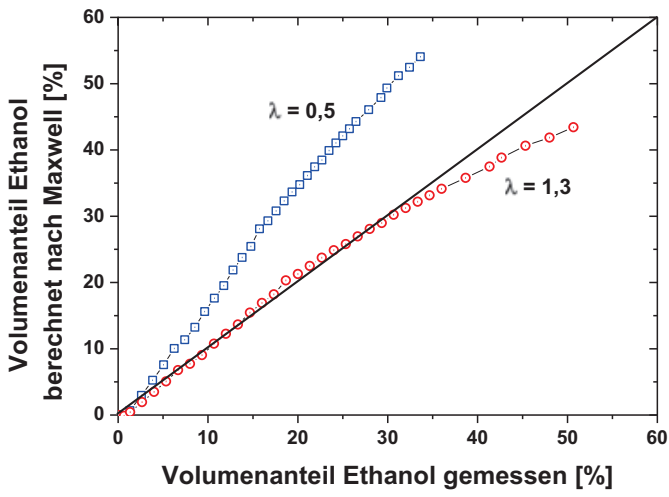

Bild 3. Vergleich des gemessenen und nach Gleichung (2) berechneten Volumenanteils

Ethanol für $\sigma_{Z} / \sigma_{T}=0,01$

Insgesamt erscheint die Ermittelung des Volumenverhältnisses von Flüssigkeitsmischungen nach Gleichung (2) problematisch, da der Geometriefaktor $\lambda$ von den Leitfähigkeiten der Komponenten abhängt und eine gute Übereinstimmung von Messung und Rechnung durch die Nichtlinearität der Gleichung (2) nur in einem begrenzten Bereich möglich ist.

\section{Messung von Flüssigkeitsmischungen mit dem Blasen - Detektor}

Zur Untersuchung der Abhängigkeit des Ausgangssignals des Blasen-Detektors von der Leitfähigkeit des Fluids wurden Versuche durchgeführt, bei denen die elektrische Leitfähigkeit von Wasser bei konstanter Temperatur durch Zugaben von Natriumchlorid verändert und dann für die gleiche Blasengröße die aus einer Zeitserie gemittelte Amplitude des Blasensignals als Funktion der Leitfähigkeit dargestellt wurde, siehe Bild 4. Das Amplitudensignal steigt insbesondere für geringe Leitfähigkeiten stark an. Wählt man jedoch einen beliebigen Arbeitspunkt und nur geringe Änderungen der Leitfähigkeit, z. B. einige 10 $\mu \mathrm{S} / \mathrm{cm}$, dann kann diese Änderung näherungsweise als linear angenommen werden.

Zur Bestimmung der Abhängigkeit des Signals des Blasen-Detektors vom Ethanol-Volumenanteil wurde mit Coomassie-Brilliant Blau R-50, $\mathrm{Fa}$. Serva, eingefärbtes Ethanol in Wasser eingeschüttet und der Zeitverlauf des Signals aufgenommen, siehe Bild 5. Nach dem Einschütten stellte sich eine stabile Ethanolschicht auf dem Wasser aus, die zu einem konstanten Anstieg des Signals führte. Nach der vollständigen Durchmischung durch Umrühren und einer Leitfähigkeitsmessung stellte sich der endgültige Wert des Signals ein. Zur Bestimmung der Kennlinie wurde jeweils das Signal des Blasen-Detektors nach der voll- 
ständigen Durchmischung $S_{\text {Mix }}$ auf das Signal des reinen Trägermaterials vor Beginn der Zumischung bezogen und als Funktion des Volumenanteils der Zumischung dargestellt. Das Bild 6 zeigt drei Messungen, bei denen Ethanol (reines Ethanol, $\sigma=5,4 \mu S / \mathrm{cm}$, und mit mit Coomassie eingefärbtes Ethanol, $\sigma=14$ $\mu \mathrm{S} / \mathrm{cm}$ ) oder hochreines Wasser mit $\sigma=1,3$ $\mu S / \mathrm{cm}$ in Wasser, $\sigma=79-82 \mu S / \mathrm{cm}$, gemischt

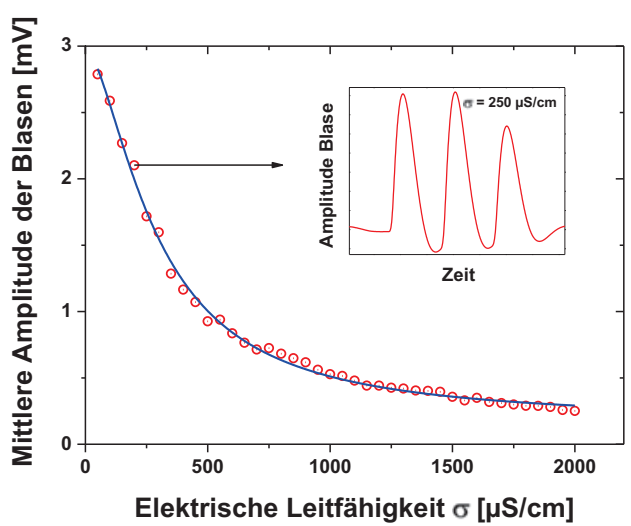

Bild 4. Mittlere Amplitude der Blasen (Blasendurchmesser etwa 2 - $3 \mathrm{~mm}$ ) als Funktion der elektrischen Leitfähigkeit der Flüssigkeit

wurden. Bei allen Kennlinien ergibt sich eine hohe Linearität mit einem Korrelationskoeffizient $r$ größer als 0,99. Es zeigt sich jedoch auch eine deutliche Abhängigkeit vom Verhältnis der Leitfähigkeiten der reinen Phasen $\sigma_{Z} / \sigma_{T}$, so dass eine Kalibrierung bei der Verwendung als Sensor notwendig ist.

Auch die Injektion von Flüssigkeiten von unten durch eine Öffnung in der Bodenplatte des Blasen-Detektors - im Gegensatz zum Einschütten von oben - kann detektiert werden. Bei der Injektion von $1 \mathrm{ml}$ Ethanol steigt die Flüssigkeit durch Auftrieb als Wolke durch die Ringelektroden und ergibt einen deutlichen Signalanstieg von $0,5-1 \mathrm{mV}$.

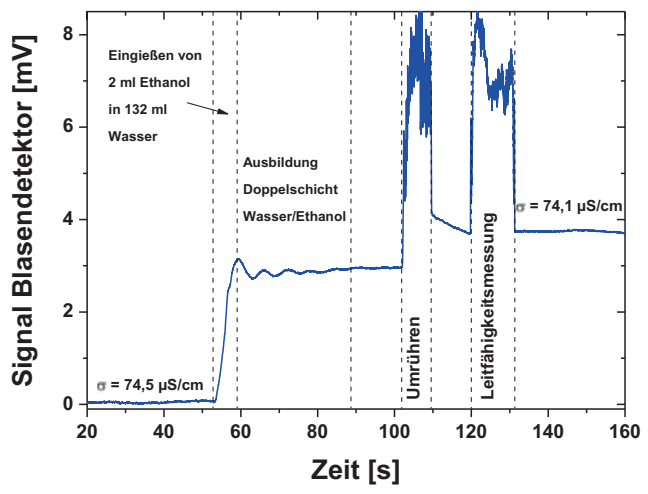

Bild 5. Zeitsignal des Blasen-Detektors während der Mischung von Ethanol in Wasser

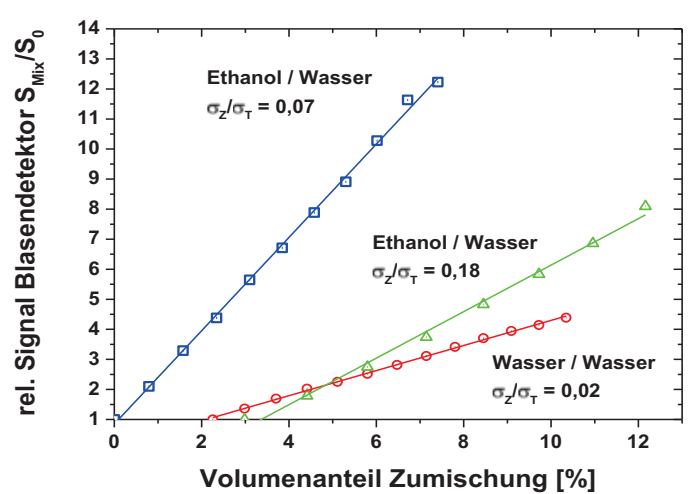

Bild 6. Relatives Signal des Blasen-Detektors (Bezug Signal ohne Zumischung) für Ethanol / Wasser - Mischungen und Reinwasser / WasserMischungen in Abhängigkeit des Volumenanteils

\section{Messung von Blasenwolken mit dem Blasen - Detektor}

Bei der Injektion von Gasströmen in Blasensäulen ist die Kenntnis des Gasanteils wichtig zur Steuerung des verfahrenstechnischen Prozesses. Die dazu eingesetzten Verfahren sind z.B. Nadelsonden-Verfahren für die örtliche Messung oder Druck-Gradienten-Verfahren, Schnellschluss-Ventile oder ImpedanzVerfahren nach Gleichung (2) zur globalen Messung [24]. Es soll im Folgenden untersucht werden, ob der Blasen-Detektor als modifiziertes Impedanzverfahren hier einen Beitrag liefern kann.

Zur Erzeugung der Blasenwolke wurde in den Boden des Blasen-Detektors ein poröser Sprudelstein eingesetzt, der an eine Schlauchoder Spritzenpumpe angeschlossen wurde, siehe Bild 6. Die mittlere Größe der Blasen war leicht von der Höhe des Durchsatzes abhängig und wird mit 0,5 - 1,0 mm abgeschätzt. Der mittlere Durchsatz wurde durch eine Volumenund Zeitmessung ermittelt. Insbesondere bei der Spritzenpumpe ergaben sich deutliche Schwankungen des Durchsatzes, sodass eine signifikante Streuung der Messergebnisse auftrat. Deshalb wurden mehrere Messserien durchgeführt. Das Bild 7 zeigt im Vergleich das gleichmäßige Zeitsignal einer Blasenwolke, die mit der Schlauchpumpe erzeugt wurde, und das Zeitsignal des Blasenstroms mit einer Spritzenpumpe.

Es ist offensichtlich, dass die Schlauchpumpe einen gleichmäßigeren Durchfluss liefert als die Spritzenpumpe, wobei die Spritzenpumpe einen eine wesentlich flexiblere Einstellung des Durchflusses ermöglicht. Zur Bestimmung des Signals des Blasen-Detektors als Funktion des Durchflusses wurde ein mittlerer Wert im Messzeitraum bestimmt und der Durchfluss aus einer Volumen- und Zeitmessung berechnet. 


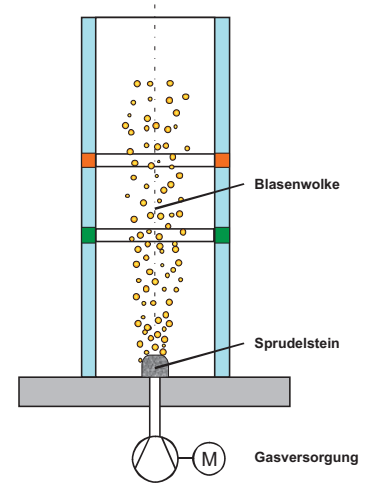

Bild 6. Aufbau zur Erzeugung und Messung von Blasenwolken mit einem Sprudelstein
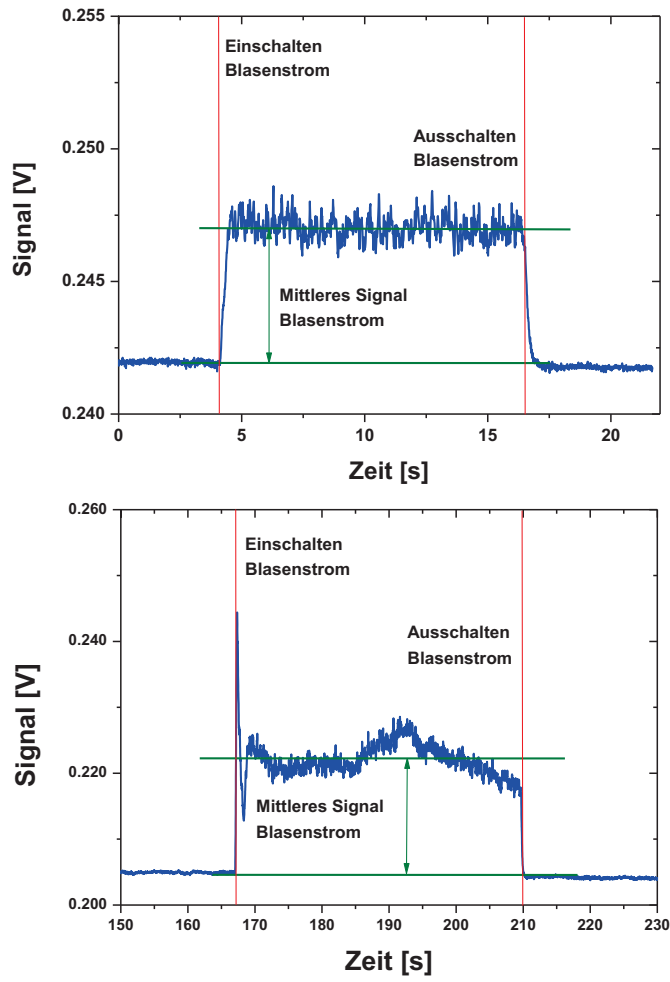

Bild 7. Zeitsignal beim Ein- und Ausschalten eines Blasenstroms, der mit einer Schlauchpumpe (oben) und einer Spritzenpumpe (unten) erzeugt wurde

Die Ungleichmäßigkeit des Messsignals und Unsicherheiten bei der Bestimmung des Volumenstroms führten zu einer deutlichen Streuung der Messwerte. Das Bild 8 zeigt deshalb den Vergleich von vier Messserien, wobei die Leitfähigkeit des Wassers etwa 50 $80 \mu \mathrm{S} / \mathrm{cm}$ betrug. Auch hier kann bei einem gleichmäßigen Blasenstrom und einer exakten Bestimmung des Durchflusses von einem linearen Verhalten ausgegangen werden. Vielmehr zeigt die Registrierung von Ungleichmäßigkeiten im Durchfluss, z.B. die Spitze beim Einsetzen des Blasenstroms der Spritzenpumpe, siehe Bild 7 unten, dass Vorgänge wie z.B. hier das plötzliche Ausbrechen des Blasenstroms nach einer

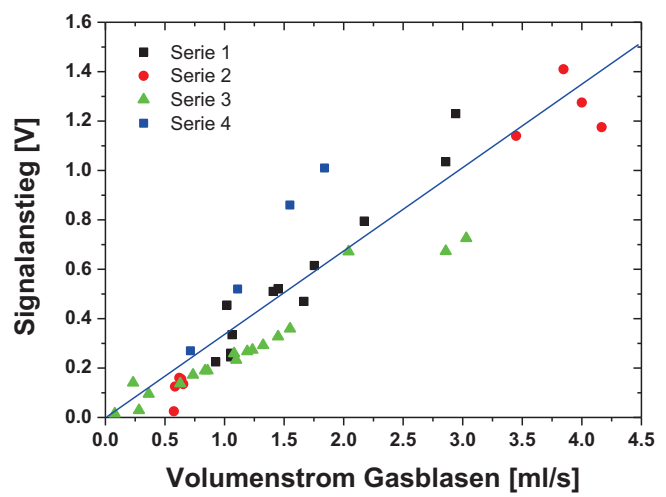

Bild 8. Signal des Blasen-Detektors bei verschiedenen Messserien mit Schlauch- und Spritzenpumpe. Leitfähigkeit des Wassers 50 $80 \mu \mathrm{S} / \mathrm{cm}$

Komprimierung des Gasvolumens in der Spritze und die Reduzierung bei Einsetzen der Gleitbewegung des Spritzenkolbens, mit einer hohen Zeitauflösung registriert werden können. Die Bestimmung des Blasen-Volumenanteils kann nur mit hohem apparativem Aufwand einigermaßen zuverlässig durchgeführt werden, z.B. Röntgenverfahren. Eine relativ einfache Berechnung des BlasenVolumenanteils kann näherungsweise durchgeführt werden mit [25]:

$$
\Phi_{\mathrm{B}}=\frac{\mathrm{V}_{\mathrm{B}}}{\mathrm{A} \cdot \mathrm{U}_{\mathrm{BW}}}
$$

Mit: $\phi_{\mathrm{B}}[-]$ : mittlerer Volumenanteil der Blasen im Wasser, $V_{B}\left[\mathrm{~mm}^{3} / \mathrm{s}\right]$ : injizierter Volumenstrom Luft, $A\left[\mathrm{~mm}^{2}\right]$ : Behälterquerschnitt, $U_{B W}[\mathrm{~mm} / \mathrm{s}]$ : Schwarmgeschwindigkeit der Blasen

Hierbei ist allerdings die exakte Bestimmung der mittleren Schwarmgeschwindigkeit aufwändig, sodass hier nur eine grobe Bestimmung durchgeführt werden konnte. Als Schwarmgeschwindigkeit der Blasen wurde etwa $125 \mathrm{~mm} / \mathrm{s}$ ermittelt, wodurch sich ein mittlerer Volumenanteil der Blasen von einigen Prozent ergibt. Damit ist gewährleistet, dass sich keine Abhängigkeit zwischen $\phi_{\mathrm{B}}$ und $\mathrm{U}_{\mathrm{BW}}$ ergibt. Diese Abhängigkeit tritt in der Regel erst bei deutlich höheren Volumenanteilen auf.

Mit einer modifizierten Gleichung auf der Basis von Gleichung (2) kann der Volumenanteil unter gewissen Voraussetzungen aus den Signalen des Blasen-Detektors ermittelt werden.

$$
\Phi_{\mathrm{B}}=\frac{\left[\frac{\mathrm{S}_{\text {Mix }}}{\mathrm{S}_{0}}-1\right] \cdot \alpha}{1+\lambda \cdot\left[\frac{\mathrm{S}_{\mathrm{Mix}}}{\mathrm{S}_{0}}\right]}
$$

Mit: $\phi_{\mathrm{B}}$ : [\%]: mittlerer Volumenanteil der Blasen, $\mathrm{S}_{\text {Mix }}, \mathrm{S}_{0}[\mathrm{~V}]$ : Signal des Blasen-Detektors mit und ohne Blasenstrom, $\lambda$ : Geometriefaktor, $\alpha$ : Korrekturfaktor 


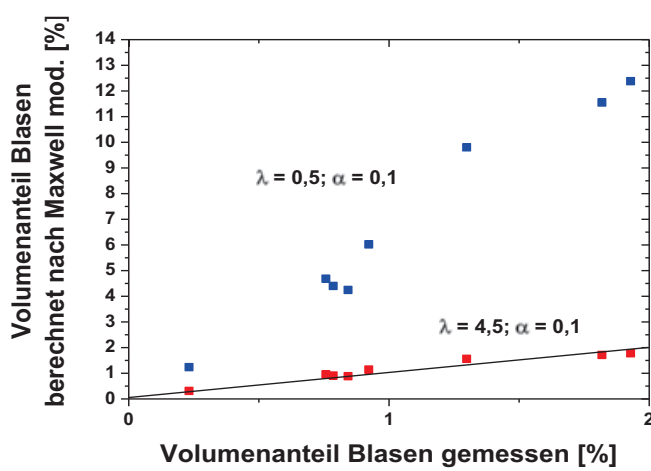

Bild 9. Vergleich der Messserie 3 aus Bild 8 mit der Gleichung (4) für $\lambda=0,5$ und 3,5 sowie $\alpha=$ 0,1 . Der gemessene Volumenanteil wurde mit $U_{B W}$ $=125 \mathrm{~mm} / \mathrm{s}$ aus Gleichung (3) ermittelt.

Als notwendige Voraussetzung muss die Ausgangsspannung des Blasen-Detektors positiv und größer als Null sein. Für eine ausgewählte Versuchsserie aus Bild 8, für die diese Voraussetzungen zutreffen, zeigt das Bild 9 , dass für $\lambda=4,5$ und $\alpha=0,1$ die Messungen sehr gut durch die Gleichung (4) beschrieben werden kann. Um eine verbesserte Gültigkeit zu erreichen muss die Elektronik so modifiziert werden, dass die oben genannten Voraussetzungen erfüllt sind.

\section{References}

[1] Crowe, C. T., 2005, Multiphase Flow Handbook, CRC Press, Boca Raton London New York.

[2] Cartellier, A., 1990, "Optical Probes for Local Void Fraction Measurements - Characterization of Performance," Rev Sci Instrum, 61(2), pp. 874886.

[3] Vagle, S., and Farmer, D. M., 1998, "A comparison of four methods for bubble size and void fraction measurements," leee J Oceanic Eng, 23(3), pp. 211-222.

[4] George, D. L., Shollenberger, K. A., Torczynski, J. R., O'Hern, T. J., and Ceccio, S. L., 2001, "Threephase material distribution measurements in a vertical flow using gamma-densitometry tomography and electrical-impedance tomography," International Journal of Multiphase Flow, 27(11), pp. 1903-1930.

[5] York, T., 2001, "Status of electrical tomography in industrial applications," J Electron Imaging, 10(3), pp. 608-619.

[6] Uesawa, S., Kaneko, A., and Abe, Y., 2012, "Measurement of void fraction in dispersed bubbly flow containing micro-bubbles with the constant electric current method," Flow Meas Instrum, 24, pp. 50-62.

[7] Yang, W. Q., 2010, "Design of electrical capacitance tomography sensors," Meas Sci Technol, 21(4).

[8] Geraets, J. J. M., and Borst, J. C., 1988, "A capacitance sensor for two-phase void fraction measurement and flow pattern identification," International Journal of Multiphase Flow, 14(3), pp. 305-320.

[9] Gerald Austen, W., and Howry, D. H., 1965, "Ultrasound as a method to detect bubbles or particulate matter in the arterial line during cardiopulmonary bypass," Journal of Surgical Research, 5(6), pp. 283-284.

[10] Abdalrahman Ahmed, M. G., Adam, A. B., and Dennis, J. O., 2009, "Capacitive Air Bubble Detector Operated at Different Frequencies for Application in Hemodialysis," Proceedings of World Academy of Science: Engineering \& Technology, 50, pp. 88-91.

[11] Sensirion, 2012, "Flow Switch and Bubble Detector LG01 for Liquids,"

[12] Nebuya, S., Noshiro, M., Brown, B. H., Smallwood, R. H., and Milnes, P., 2004, "Estimation of the size of air emboli detectable by electrical impedance measurement," Med Biol Eng Comput, 42(1), pp. 142-144.

[13] von der Emde, G., Amey, M., Engelmann, J., Fetz, S., Folde, C., Hollmann, M., Metzen, M., and Pusch, R., 2008, "Active electrolocation in Gnathonemus petersii: Behaviour, sensory performance, and receptor systems," J Physiology-Paris, 102(4-6), pp. 279-290.

[14] von der Emde, G., Behr, K., Bouton, B., Engelmann, J., Fetz, S., and Folde, C., 2010, "3Dimensional Scene Perception during Active Electrolocation in a Weakly Electric Pulse Fish," Frontiers in behavioral neuroscience, 4, p. 26.

[15] Mayekar, K., Damalla, D., Gottwald, M., Bousack, H., and von der Emde, G., 2012, "A Multi-electrode Biomimetic Electrolocation Sensor," Bioinspiration, Biomimetics, and Bioreplication 2012, 8339.

[16] Bousack, H., Mayekar, K., Von der Emde, G., and Zhou, R., 2012, "Blasendetektor," Patentanmeldung Forschungszentrum Jülich

[17] Maxwell, J. C., 1904, A Treatise on Electricity and Magnetism, Clarendon Press, Oxford.

[18] Hewitt, G. F., 1978, Measurement of two phase flow parameters, Academic Press

[19] George, D. L., Torczynski, J. R., Shollenberger, K. A., O'Hern, T. J., and Ceccio, S. L., 2000, "Validation of electrical-impedance tomography for measurements of material distribution in twophase flows," International Journal of Multiphase Flow, 26(4), pp. 549-581.

[20] Fricke, H., 1924, "A Mathematical Treatment of the Electric Conductivity and Capacity of Disperse Systems I. The Electric Conductivity of a Suspension of Homogeneous Spheroids," Physical Review, 24(5), pp. 575-587.

[21] Ninnis, R., 1991, "Theory of void fraction sensors."

[22] Bruggeman, D. A. G., 1935, "Berechnung verschiedener physikalischer Konstanten von heterogenen Substanzen. I. Dielektrizitätskonstanten und Leitfähigkeiten der Mischkörper aus isotropen Substanzen," Annalen der Physik, 416(7), pp. 636-664.

[23] Bottcher, C. J. F., 1945, "The Dielectric Constant of Crystalline Powders," Recl Trav Chim Pay B, 64(1-2), pp. 47-51.

[24] Chaouki, J., Larachi, F., and Duduković, M. P., 1997, Non-Invasive Monitoring of Multiphase Flows, Elsevier Science BV, Amsterdam

[25] Chmiel, H., 2011, Bioprozesstechnik, Spektrum Akademischer Verlag Heidelberg. 